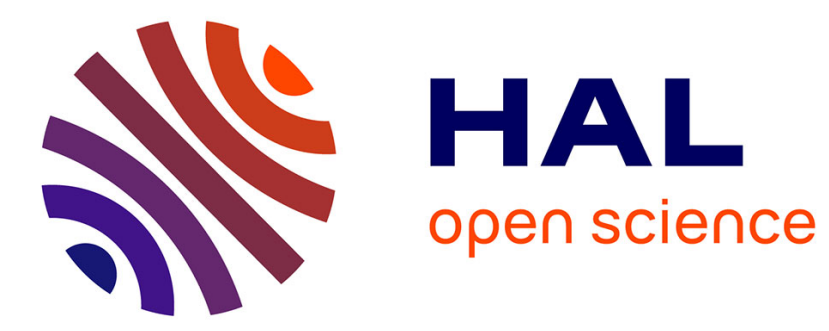

\title{
Dynamics of evanescent matter waves in negative-index media
}

\author{
M Hamamda, V Bocvarski, F Perales, J Baudon, G Dutier, C Mainos, M \\ Boustimi, M Ducloy
}

\section{- To cite this version:}

M Hamamda, V Bocvarski, F Perales, J Baudon, G Dutier, et al.. Dynamics of evanescent matter waves in negative-index media. Journal of Physics B: Atomic, Molecular and Optical Physics, 2010, 43 (21), pp.215301. 10.1088/0953-4075/43/21/215301 . hal-00597869

\section{HAL Id: hal-00597869 \\ https://hal.science/hal-00597869}

Submitted on 2 Jun 2011

HAL is a multi-disciplinary open access archive for the deposit and dissemination of scientific research documents, whether they are published or not. The documents may come from teaching and research institutions in France or abroad, or from public or private research centers.
L'archive ouverte pluridisciplinaire HAL, est destinée au dépôt et à la diffusion de documents scientifiques de niveau recherche, publiés ou non, émanant des établissements d'enseignement et de recherche français ou étrangers, des laboratoires publics ou privés. 


\title{
Dynamics of evanescent matter waves in negative-index media
}

\author{
M. Hamamda, V. Bocvarski ${ }^{1}$, F. Perales , J. Baudon", G. Dutier, C. Mainos, \\ M. Boustimi ${ }^{2}$ and M. Ducloy \\ Laboratoire de Physique des Lasers, CNRS - UMR 7538, Université Paris 13, \\ 99 Av. J.B. Clément, 93430-Villetaneuse, France \\ $\left({ }^{1}\right)$ Permanent address: Institute of Physics-Belgrade; University of Belgrade, Pregrevica 18, \\ PO Box 57, 11080-Zemun (Serbia) \\ $\left(^{2}\right)$ Permanent address: Department of Physics, Umm Al-Qura University, \\ PO box 715 - Makkah, Saudi Arabia \\ * Corresponding author: jacques.baudon@univ-paris13.fr \\ PACS numbers: \\ 03.75.-b Matter Waves \\ 03.75.Be Atom and Neutron Optics \\ 37.10.Gh Atom Traps and Guides \\ 42.25.-p Wave Optics
}

\begin{abstract}
Semi-evanescent and evanescent matter-waves produced by an atom wave packet impinging a repulsive barrier can be back-refracted and reconstructed by the application of negative-index "comoving" potential pulses. One shows that those collapses and revivals generate a matter wave confined on both sides of the barrier border ("surface matter wave") and should be observable via the retardation of atom reflection from the barrier interface. This property, joined to the possibility recently demonstrated of inducing negative refraction of atom waves, makes such potentials a matter-wave counterpart of negative-index materials or "meta materials" well-known in light optics.
\end{abstract}


With the fast development of matter-wave optics, many of the functions previously operated in light optics have been realised: atom diffraction and mirrors, beam splitters, atom lasers, atom holography, quantum reflection, etc. [1]. Specific characters of those processes originate in the properties of the associated particle: non-zero atom mass, vacuum dispersion for the "de Broglie" waves (implying longitudinal wave packet spreading), scalar character of the atomic wave function, influence of the internal atomic degrees of freedom, etc. Along this viewpoint, novel areas in the field of atom optics are presently explored, including e.g., the devising of non-diffracting atom nano-beams via a specially designed transverse SternGerlach interferometer [2]. Recently, we have proposed to extend the concept of "metaoptics" (known as negative index materials, NIM-s, in light optics [3-4]) to matter waves [5]. The main specificity of NIM-s for atom optics is their transient character, linked to the fact that for matter waves the reversal of atom group velocity is operated and can be only transient - contrary to the equivalent process in light optics where the phase velocity is reversed. In [5], it was proposed to use a "comoving" potential pulse [6] to reverse the atom group velocity. Previously only the trajectory of the wave packet centre has been investigated and a negative refraction predicted. In this letter, we examine the behaviour of the wave packet itself in negative-index media and analyse its dynamics both in free space and classically forbidden regions (evanescent or semi-evanescent matter wave packets). The dynamics of matter waves impinging a static potential barrier is strongly altered by the comoving potential. In particular, we investigate here the possibility of evanescent wave-packet engineering and predict the generation of atom reflection replicas as well as matter waves confined in the vicinity of the barrier edge.

It is commonly admitted that, in the semi-classical regime, light and matter waves involving a single direction of space (e.g. x), behave identically provided they are monochromatic, i.e. provided they involve a single value of the wave number k (or particle's energy). For both kinds of waves, the evanescent wave appears, at a normal incidence with respect to a planar interface, as soon as the refractive index of the second medium is imaginary. This means for light a conducting medium (metal, plasma). For particles, a constant potential energy barrier $\mathrm{V}_{0}$, extending from $\mathrm{x}=0$ to $\mathrm{x}$ infinite, higher than the initial kinetic energy $\mathrm{E}_{0}$ of the particle, or, in the case of a wave packet, higher than the kineticenergy distribution, provides an imaginary index since the index for matter waves in the region $\mathrm{x}>0$ is $\mathrm{n}=\left(1-\mathrm{V}_{0} / \mathrm{E}_{0}\right)^{1 / 2}$. In a $2 \mathrm{D}$ geometry $(\mathrm{x}, \mathrm{z})$ with the same potential barrier, the motion along $\mathrm{z}$ is free, which leads to a total reflection in the $\mathrm{x}<0$ half plane and to an evanescent wave in the $\mathrm{x}>0$ side. The corresponding index is static. As shown in [5], a negative index for matter waves is necessarily a transient process, thus needing incident wave packets, i.e. some k-momentum distribution. As a consequence, light and matter evanescent waves behave differently. While light evanescent waves are factorized in $\mathrm{x}$ and $\mathrm{t}$ (at least in a non-dispersive medium), such a factorisation does not hold for evanescent matter waves, simply because of intrinsic vacuum dispersion: the time dependence is in exp $\left[-\mathrm{i} \hbar \mathrm{k}^{2} \mathrm{t} /(2 \mathrm{~m})\right]$, instead of $\exp [-\mathrm{i} \mathrm{ck} t]$, where $\mathrm{m}$ is the atomic mass and $\mathrm{c}$ the light velocity. The existence of evanescent matter waves is one of the fundamental processes introduced by quantum mechanics and has many outstanding consequences, mainly explored, up to now, for electrons (like electron tunnelling, as in tunnelling microscopy [7], or Josephson Effect in superconductivity [8]). For atomic systems, it has not been much studied, except in molecular spectroscopy [9], collision physics [10] and more recently cold atom physics [11], where evanescent waves are commonly involved (e.g. quasi-bound states and resonances of various types). 
The evolution in $\mathrm{x}$ and $\mathrm{t}$ of a partially evanescent matter-wave packet, experiencing a pulse of comoving potential, will be described in the following. This comoving potential has the general form:

$$
\mathrm{V}(\mathrm{t}, \mathrm{x})=\mathrm{s}(\mathrm{t}) \cos (2 \pi \mathrm{x} / \Lambda)
$$

where $\mathrm{s}(\mathrm{t})$ is a real signal restricted to a finite time interval $\left(0, \tau_{1}\right)$ and $\Lambda$ is the spatial period. To really construct a "comoving" potential, it is necessary that the spectrum $H(v)$ of $s(t)$ contains a frequency matching the atomic velocity $\left(v_{0}=\hbar \mathrm{k}_{0} /(\mathrm{m} \Lambda), \mathrm{k}_{0}\right.$ being the central atomic momentum. A simple - while not fully optimized - form is:

$$
\begin{aligned}
& \mathrm{s}(\mathrm{t})=\mathrm{C} \mathrm{e}^{+20 \mathrm{t} / \tau} \text { for } \mathrm{t} \text { in the interval }[-\infty, 0] \\
& \mathrm{s}(\mathrm{t})=\mathrm{C} \varepsilon^{2}(\mathrm{t}+\varepsilon)^{-2} \mathrm{e}^{-\mathrm{t} / \tau} \quad \text { for } 0 \leq \mathrm{t} \leq \tau_{1} ;=0 \text { for } \mathrm{t}>\tau_{1}
\end{aligned}
$$

where $\mathrm{C}, \varepsilon, \tau, \tau_{1}$ are constants. We consider here atoms the spin of which is $\mathrm{J}=2$, like argon metastable atoms $\left(A{ }^{*}{ }^{3} \mathrm{P}_{2}\right.$ ) having a velocity along $\mathrm{x}$ axis of $4 \mathrm{~m} / \mathrm{s}$ (de Broglie wavelength $\lambda_{\mathrm{dB}} \approx 2.8 \mathrm{~nm}$, central momentum value $\mathrm{k}_{0}=2.24410^{9} \mathrm{~m}^{-1}$, momentum dispersion $\delta \mathrm{k}=0.005$ $\mathrm{k}_{0}$ ), polarized in the $\mathrm{M}=+2$ Zeeman sublevel. For such atoms, a magnetic potential can be used, with $C=g_{L} \mu_{B} B_{\max } M$, where $g_{L}$ is the Landé factor, $\mu_{B}$ the Bohr magneton, $B_{\max }$ the maximum value of the magnetic field magnitude and $M$ the magnetic quantum number. Evanescent wave packets appear when $\mathrm{k}<\mathrm{k}_{0}$ within a repulsive potential barrier of momentum height $\mathrm{a}=\mathrm{k}_{0}$ (this corresponds to a magnetic field of 127 Gauss). For Ar* atoms, the repulsive potential barrier can be created using a static magnetic field $\mathrm{B}_{0}$ in the half-space $\mathrm{x}>0$ (see figure 1).

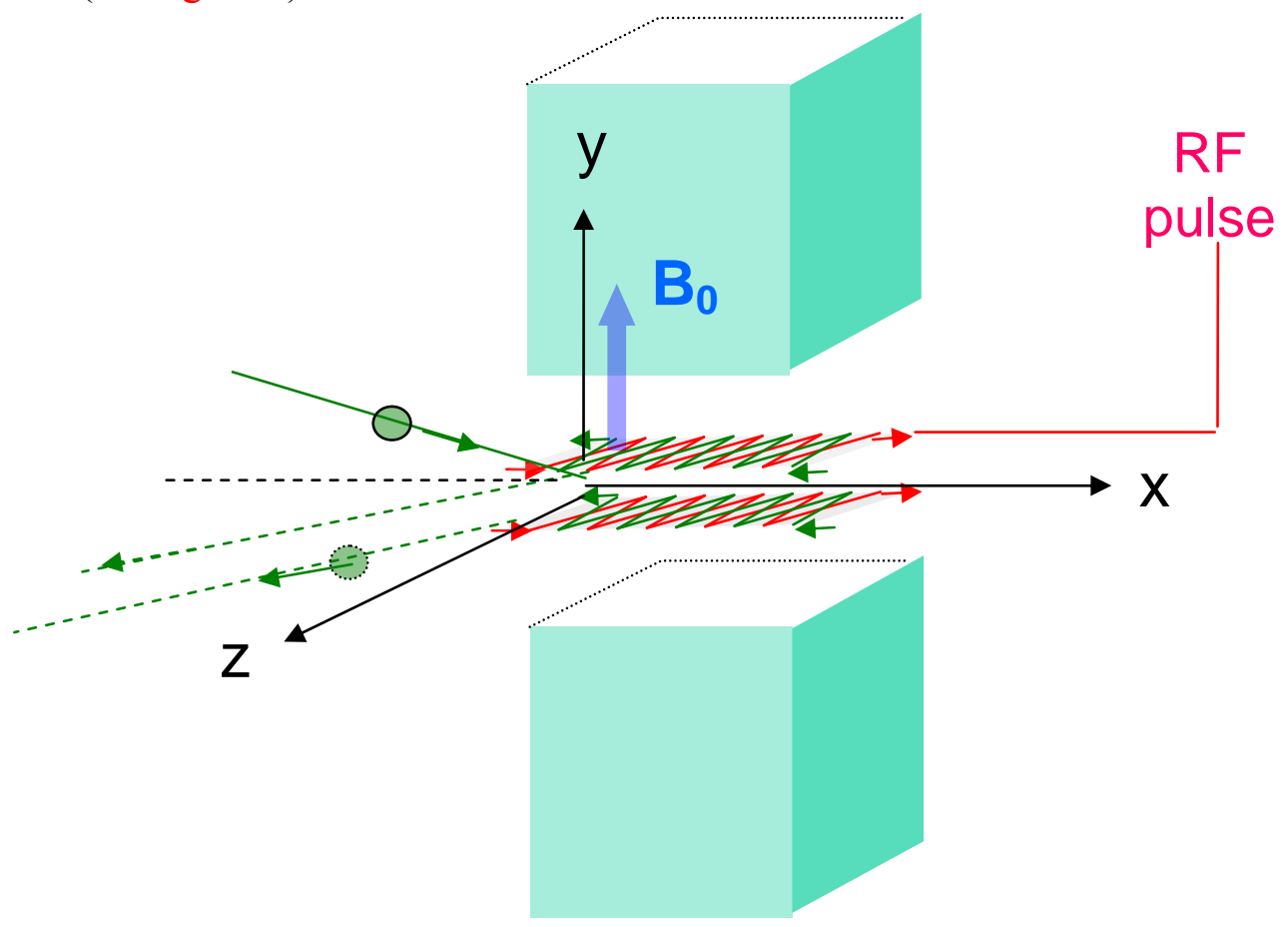

Figure 1. Principle scheme of an experiment on evanescent and semi-evanescent wave packets submitted to comoving magnetic potential pulses (see text). A static potential barrier is generated by the static magnetic field $\mathbf{B}_{0}$. Potential pulses, comoving in the $\mathrm{x}$ direction, are produced by a generator ("RF pulse"). The incident wave packet propagates in plane $(\mathrm{x}, \mathrm{z})$. The reflected wave packets propagate in a direction of the $(\mathrm{x}, \mathrm{z})$ plane, symmetric of the incident direction with respect to the $\mathrm{x}$ axis. 
The magnetic potential actually takes the form (1a) - plus a constant within the barrier -, provided that a given value of $\mathrm{M}$, e.g. +2 , referred to the comoving field axis (y) is prepared and maintained, which implies the presence of a tiny static field ( $b$ of a few Gauss) on both sides of the barrier edge. The easiest way to realize this condition is to use a collinear configuration, $i$. e. to make $\underline{B}_{0}$ parallel to y axis. In fact, the static field cannot be abruptly increased from zero (or b) up to $\mathrm{B}_{0}$, which means that the barrier edge has necessarily a finite thickness. It should be possible to analyse in some detail the effect that it causes to the reflection/transmission at the barrier edge (e.g. in the case of a linear increase, by use of a combination of Airy functions). Nevertheless, provided that the thickness is less than the spatial period $\Lambda$, and owing to the fact that the comoving field starts at a given time, this thickness is expected to be of little importance on the dynamics of incident, reflected and transmitted wave packets.

We are dealing here with three wave functions, namely $\psi_{\mathrm{i}}(\mathrm{t}, \mathrm{x})$, the incident wave packet propagating from $\mathrm{x}=-\infty$ to $\mathrm{x}=0, \psi_{\mathrm{r}}(\mathrm{t}, \mathrm{x})$, the reflected wave packet propagating backwards from $\mathrm{x}=0$ to $\mathrm{x}=-\infty$, and the transmitted evanescent wave $\psi_{\mathrm{tr}}(\mathrm{t}, \mathrm{x})$ in the region $\mathrm{x}$ $>0$. For a specific value of the wave number $\mathrm{k}$, i.e. a specific value $\mathrm{E}$ of the total incident energy, the three wave functions have the same time-dependence, in exp $\left[-i \hbar k^{2} t /(2 \mathrm{~m})\right]$. In the absence of comoving field, the conservation of the probability flux leads to the usual reflection and transmission factors (in amplitude):

$$
\mathrm{R}(\mathrm{k})=\frac{\mathrm{k}-\mathrm{i} \kappa}{\mathrm{k}+\mathrm{i} \kappa} ; \mathrm{T}(\mathrm{k})=\frac{2 \mathrm{k}}{\mathrm{k}+\mathrm{i} \kappa}
$$

, with $\kappa=\sqrt{\mathrm{a}^{2}-\mathrm{k}^{2}}$ and $\mathrm{a}^{2}=\left(2 \mathrm{~m} / \hbar^{2}\right) \mathrm{V}_{0}>\mathrm{k}^{2}$. As expected, the evanescent wave does not carry any probability flux then $|R|=1$. Similar expressions of $R$ and $T$ are obtained for $k \geq a$ (partial propagation through the barrier), by replacing iк by $\mathrm{k}^{\prime}=\sqrt{\mathrm{k}^{2}-\mathrm{a}^{2}}$. Let $\Psi_{\text {tro }}(\mathrm{t}, \mathrm{x})$ be the solution for $\mathrm{x}>0$ in absence of comoving potential, that is (up to a multiplicative constant):

$$
\psi_{\operatorname{tr} 0}(\mathrm{t}, \mathrm{x})=\int_{-\infty}^{+\infty} \mathrm{d} \mathrm{k} T(\mathrm{k}) \rho(\mathrm{k}) \phi_{\operatorname{tr} 0}(\mathrm{t}, \mathrm{x}, \mathrm{k})
$$

$\rho(\mathrm{k})$ being the momentum distribution and $\phi_{\mathrm{tr} 0}$ the evanescent $\mathrm{k}-$ spectral component:

$$
\phi_{\text {tr } 0}(\mathrm{t}, \mathrm{x}, \mathrm{k})=\exp (-\kappa \mathrm{x}) \exp \left[-\mathrm{i} \hbar \mathrm{k}^{2} \mathrm{t} /(2 \mathrm{~m})\right]
$$

Expansions similar to (4a) are obtained for incident and reflected waves, namely:

$$
\begin{aligned}
& \psi_{\mathrm{i} 0}(\mathrm{t}, \mathrm{x})=\int_{-\infty}^{+\infty} \mathrm{d} \mathrm{k} \rho(\mathrm{k}) \phi_{\mathrm{i} 0}(\mathrm{t}, \mathrm{x}, \mathrm{k}) \\
& \psi_{\mathrm{r} 0}(\mathrm{t}, \mathrm{x})=\int_{-\infty}^{+\infty} \mathrm{d} \mathrm{k} R(\mathrm{k}) \rho(\mathrm{k}) \phi_{\mathrm{r} 0}(\mathrm{t}, \mathrm{x}, \mathrm{k})
\end{aligned}
$$

with spectral components :

$$
\begin{aligned}
& \phi_{\mathrm{i} 0}(\mathrm{k}, \mathrm{t}, \mathrm{x})=\exp (\mathrm{ikx}) \exp \left[-\mathrm{i} \hbar \mathrm{k}^{2} \mathrm{t} /(2 \mathrm{~m})\right] \\
& \phi_{\mathrm{r} 0}(\mathrm{k}, \mathrm{t}, \mathrm{x})=\exp (-\mathrm{i} \mathrm{kx}) \exp \left[-\mathrm{i} \hbar \mathrm{k}^{2} \mathrm{t} /(2 \mathrm{~m})\right]
\end{aligned}
$$


Let us assume that the effect of the comoving potential pulse on the spectral components of the wave packets consists of simple multiplicative factors:

$$
\phi_{\mathrm{i}, \mathrm{r}, \mathrm{tr}}=\phi_{\mathrm{i} 0, \mathrm{r} 0, \text { tr } 0} \mathrm{f}_{\mathrm{i}, \mathrm{r}, \mathrm{tr}}(\mathrm{k}, \mathrm{t}, \mathrm{x})
$$

The time dependent Schrödinger equation in half-space $\mathrm{x}>0$ is:

$$
\mathrm{i} \hbar \partial_{\mathrm{t}} \Psi_{\mathrm{tr}}=-\frac{\hbar^{2}}{2 \mathrm{~m}} \partial_{\mathrm{x}}^{2} \Psi_{\mathrm{tr}}+\left[\mathrm{V}_{0}+\mathrm{V}(\mathrm{t}, \mathrm{x})\right] \Psi_{\mathrm{tr}}
$$

whereas in "free" half-space $\mathrm{x}<0$, it is :

$$
\mathrm{i} \hbar \partial_{\mathrm{t}} \Psi_{\mathrm{i}, \mathrm{r}}=-\frac{\hbar^{2}}{2 \mathrm{~m}} \partial_{\mathrm{x}}^{2} \Psi_{\mathrm{i}, \mathrm{r}}+\mathrm{V}(\mathrm{t}, \mathrm{x}) \Psi_{\mathrm{i}, \mathrm{r}}
$$

Provided that the "perturbation factors" $f$ evolve, as functions of $\mathrm{x}$, much slower than the $\phi_{0}-\mathrm{s}$, then the terms in $\partial_{\mathrm{x}}{ }^{2} \mathrm{f}$ can be neglected with respect to $\kappa \partial_{\mathrm{x}} \mathrm{f}$ or $\mathrm{k} \partial_{\mathrm{x}} \mathrm{f}$. The validity of this latter approximation will be verified a posteriori. It leads for the f-s to the equations:

$$
\begin{aligned}
& i \hbar \partial_{\mathrm{t}} \mathrm{f}_{\text {tr }}=+\frac{\hbar^{2}}{\mathrm{~m}} \kappa \partial_{\mathrm{x}} \mathrm{f}_{\mathrm{tr}}+\mathrm{Vf_{ \text {tr } }} \\
& \mathrm{i} \hbar \partial_{\mathrm{t}} \mathrm{f}_{\mathrm{i}, \mathrm{r}}=+\frac{\hbar^{2}}{\mathrm{~m}} \mathrm{ik} \partial_{\mathrm{x}} \mathrm{f}_{\mathrm{i}, \mathrm{r}}+\mathrm{Vf_{i,r }}
\end{aligned}
$$

Let us define two new variables, in half-space $\mathrm{x}>0$ :

$$
\mathrm{u}=\mathrm{t}+\mathrm{i} \frac{\mathrm{m}}{\hbar \kappa} \mathrm{x} ; \mathrm{v}=\mathrm{t}-\mathrm{i} \frac{\mathrm{m}}{\hbar \kappa} \mathrm{x}
$$

and similarly in "free" half-space $\mathrm{x}<0$ :

$$
\mathrm{u}_{0}=\mathrm{t}+\frac{\mathrm{m}}{\hbar \mathrm{k}} \mathrm{x} ; \mathrm{v}_{0}=\mathrm{t}-\frac{\mathrm{m}}{\hbar \mathrm{k}} \mathrm{x}
$$

Using (9a) it is readily verified that $\left(\partial_{t}+\frac{i \hbar \kappa}{m} \partial_{x}\right)=2 \partial_{v}$. Then, using variables $u, v$, eq. (9a) becomes:

$$
\frac{\partial_{\mathrm{v}} \mathrm{f}_{\mathrm{tr}}}{\mathrm{f}_{\mathrm{tr}}}=-\frac{\mathrm{i}}{2 \hbar} \mathcal{V}(\mathrm{u}, \mathrm{v})
$$

where $\mathcal{V}(\mathrm{u}, \mathrm{v})$ is the comoving potential expressed as a function of $\mathrm{u}$ and $\mathrm{v}$.

Setting $\mathrm{f}_{\mathrm{tr}}=\exp \left[\mathrm{i} \varphi_{\mathrm{tr}}(\mathrm{u}, \mathrm{v})\right]$, one gets from (11), $\partial_{\mathrm{v}} \varphi_{\mathrm{tr}}=-\mathcal{V} /(2 \hbar)$ and finally: 


$$
\varphi_{\text {tr }}(\mathrm{u}, \mathrm{v})=-\frac{1}{2 \hbar} \int^{\mathrm{v}} \mathrm{dv}^{\prime} \mathcal{V}\left(\mathrm{u}, \mathrm{v}^{\prime}\right)+\mathrm{F}_{\mathrm{tr}}(\mathrm{u})
$$

where $F_{t r}(u)$ is an arbitrary function of $u$.

For a comoving potential of the form $(1 \mathrm{a}, \mathrm{b})$, setting at a given value of $u, v^{\prime}=2 t^{\prime}-u, E q$. (12) yields:

$$
\varphi_{\mathrm{tr}}=-\frac{1}{\hbar} \int_{0}^{(\mathrm{t})} \mathrm{dt}^{\prime} \mathrm{s}\left(\mathrm{t}^{\prime}\right) \cos \left[\frac{2 \pi}{\Lambda} \frac{\mathrm{i} \hbar \kappa}{\mathrm{m}}\left(\mathrm{u}-\mathrm{t}^{\prime}\right)\right]+\mathrm{F}_{\mathrm{tr}}(\mathrm{u})
$$

where the upper bound is $(\mathrm{t})=\operatorname{Min}\left[\mathrm{t}, \tau_{1}\right]$. Finally, the phase shift $\varphi_{\text {tr }}$ (a complex number in general) depends on 3 independent variables, $\mathrm{k}$ (via $\mathrm{\kappa})$, $\mathrm{t}$ and $\mathrm{x}$ (via $\mathrm{u}$ ).

A similar treatment can be carried out for incident and reflected waves in the free halfspace, by setting $f_{i}=\exp \left[i \varphi_{i}\left(u_{0}\right)\right]$ and $f_{r}=\exp \left[i \varphi_{r}\left(v_{0}\right)\right]$. One readily gets:

$$
\varphi_{\mathrm{i}}=-\frac{1}{\hbar} \int_{0}^{(\mathrm{t})} \mathrm{dt}^{\prime} \mathrm{s}\left(\mathrm{t}^{\prime}\right) \cos \left[\frac{2 \pi}{\Lambda} \frac{\hbar \mathrm{k}}{\mathrm{m}}\left(\mathrm{u}_{0}-\mathrm{t}^{\prime}\right)\right]+\mathrm{F}_{\mathrm{i}}\left(\mathrm{u}_{0}\right)
$$

and a similar expression for $\varphi_{\mathrm{r}}, \mathrm{u}_{0}$ being replaced by $\mathrm{v}_{0}$. The arbitrariness in the choice of the additional functions $\mathrm{F}_{\mathrm{i}, \mathrm{r}}$, tr corresponds to all possible constraints applicable to the incident, reflected and transmitted waves. To define them we have to specify the problem under consideration, namely at infinite negative values of $\mathrm{x}$ and $\mathrm{t}$, a well defined and unperturbed incoming incident wave packet impinging the barrier. Under such a condition, $F_{i}$ is a constant, which can be assumed to be 0 .

The continuity conditions at the barrier edge $(\mathrm{x}=0)$ also hold for $\phi_{\mathrm{i}}, \phi_{\mathrm{r}}$ and $\phi_{\mathrm{tr}}$. The continuity condition for the modified wave functions is :

$$
f_{i}+R f_{r}=T f_{\text {tr }}
$$

where $f_{i}, f_{r}, f_{\text {tr }}$ are taken at $x=0$. The continuity condition of the $x$-derivative at $x=0,\left[\partial_{x}\right]_{x=0}$ being noted ('), is:

$$
i k f_{i}+f_{i}{ }^{\prime}+R\left(-i k f_{r}+f_{r}{ }^{\prime}\right)=T\left(-\kappa f_{\text {tr }}+f_{\text {tr }}{ }^{\prime}\right)
$$

It can be noted that the previous expressions of $\varphi_{\mathrm{i}}$ (eq. 13b) and $\varphi_{\mathrm{r}}$ become identical at $\mathrm{x}=0$ since at that point $u_{0}=v_{0}$. It then appears as a reasonable assumption to impose $\varphi_{r}=\varphi_{i}$, i.e. $F_{r}$ $=F_{i}=0$. Under this condition, (i) one readily gets from (14) that, at $x=0, \varphi_{\text {tr }}=\varphi_{\mathrm{i}}=\varphi_{\mathrm{r}}$, from which the additional function $F_{t r}(u)$ can be deduced; (ii) eq. 15 becomes $\left(f_{i}+R_{r}-T f_{t r}\right)^{\prime}=0$.

At this point, it is interesting to compare these expressions of the $\varphi$-s to that of the phase shift - let us call it $\varphi_{\text {pro }}$ - previously used in the case of a purely propagating matter wave (cf. Ref. [5], Eq. 8):

$$
\varphi_{\text {pro }}(\mathrm{k}, \mathrm{t})=-\frac{1}{\hbar} \int_{0}^{\mathrm{t}} \mathrm{dt} \mathrm{t}^{\prime} \mathrm{s}\left(\mathrm{t}^{\prime}\right) \cos \left[\frac{2 \pi \hbar \mathrm{k}}{\mathrm{m} \Lambda} \mathrm{t}^{\prime}\right]
$$


The integral part in expressions (13a) becomes identical to (16) when iא is replaced by $\mathrm{k}$, provided that $|\mathrm{u}|$ can be considered as much smaller than $|\mathrm{v}|$. This also readily holds for $\varphi_{\mathrm{i}, \mathrm{r}}$. In other words, this equivalence holds provided that (respectively for incident and reflected waves) $\left|\mathrm{t} \mp \frac{\mathrm{m}}{\hbar \mathrm{k}} \mathrm{x}\right|<<\left|\mathrm{t} \pm \frac{\mathrm{m}}{\hbar \mathrm{k}} \mathrm{x}\right|$. This means that, in the case of propagation, all relevant values of $t$ and $x$ are close to those related to the propagation of the wave packet centre. Such an approximation, possibly valid for the incident and reflected wave packets, is clearly not valid for an evanescent wave packet.

In the following calculations, time parameters in $\mathrm{s}(\mathrm{t})$ [eq. (1b)] have been chosen to be adapted to typical characteristics of the wave packets in the region $\mathrm{x}>0$. In the present case we take $\varepsilon=7.4 \mu \mathrm{s}, \tau=0.37 \mu \mathrm{s}, \tau_{1}=0.6$ or $1.10 \mu \mathrm{s}$. The spatial period is $\Lambda=2$ or $3 \mu \mathrm{m}$ [12]. The effect of the comoving pulse on the wave functions $\psi_{\mathrm{i}}, \psi_{\mathrm{r}}, \psi_{\mathrm{tr}}$ is simply derived by incorporating the factors exp $\left(i \varphi_{i, r}\right.$, tr $)$ into the expansions of these wave functions over $\mathrm{k}$. At this point, let us examine the validity of approximation used previously to get the simplified equations $(9 \mathrm{a}, \mathrm{b})$. It turns out that, with the parameters used here, this approximation is quite good in the free half-space. Within the potential barrier $(\mathrm{x}>0)$, it becomes rather poor when the original comoving potential in $\cos (2 \pi \mathrm{x} / \Lambda)$ is used, in the sense that the ratio $\mid \kappa \partial_{\mathrm{x}}$ $\mathrm{f}_{\text {tr }}|/| \partial_{\mathrm{x}}{ }^{2} \mathrm{f}_{\text {tr }} \mid$, which is zero for $\kappa=0\left(\mathrm{k}=\mathrm{k}_{0}\right)$, becomes larger than 1 only when $\left|\mathrm{k}-\mathrm{k}_{0}\right|>$ $\delta \mathrm{k}$. The reason is that such a potential starts too abruptly at $\mathrm{x}=0$. The situation is greatly improved by slightly shifting the spatial dependence into, for instance, $\cos (2 \pi x / \Lambda-\pi / 10)$, which should be easily realized in an experiment. In such a case the ratio is larger than 10 as soon as $\left|\mathrm{k}-\mathrm{k}_{0}\right|>\delta \mathrm{k} / 20$. In other words, only a narrow central slice of the spectrum violates the approximation. It is expected - and it has been verified - to be of little importance in so far as it involves large distances from the barrier edge $(x>20 / \delta k=1.57 \mu \mathrm{m})$. This modified spatial dependence has been adopted for semi-evanescent wave packets. For almost purely evanescent ones (i.e. $\mathrm{a} \geq 1.01 \mathrm{k}_{0}$ ), this correction is not useful since the questionable part of the spectrum $(\kappa \approx 0)$ corresponds to very low values of $\left|\psi_{\text {tr }}\right|$.

The profiles in $\mathrm{t}$ and $\mathrm{x}$ of the squared modulus $\left|\psi_{\mathrm{tr} 0}\right|^{2}$ of unperturbed semi-evanescent $\left(\mathrm{a} \leq \mathrm{k}_{0}\right)$ and almost purely evanescent $\left(\mathrm{a}>\mathrm{k}_{0}\right)$ waves are shown in figure 2.

(a)

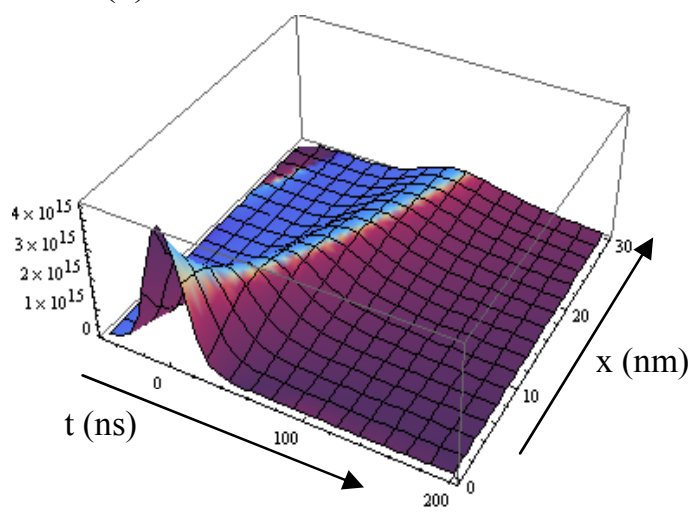

(b)

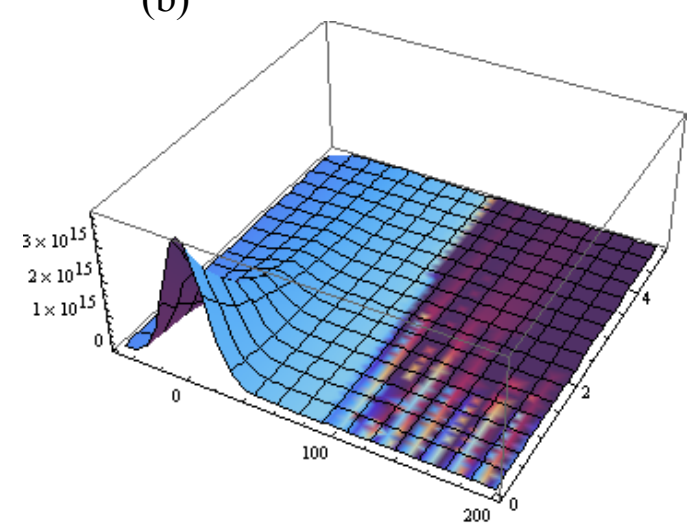

Figure 2. Profile in $t$ and $x$ of the squared modulus $\left|\psi_{\text {tro }}\right|^{2}$ of the unperturbed semi-evanescent wave function. (a) The height a (in momentum) of the potential barrier is chosen equal to central value $\mathrm{k}_{0}$ of the incident momentum distribution. At $\mathrm{x}=0$, the time dependence is Gaussian. For $\mathrm{x}>0$, one observes as expected the decay characteristic of the evanescent part together with a partial wave packet propagating at a positive group velocity and broadening in time. (b) Same as (a) for an almost pure evanescent wave $\left(\mathrm{a}=1.1 \mathrm{k}_{0}\right)$. No more propagation is seen. 
In a first step, we examine the effect of the comoving potential pulse in the semievanescent case. Figure 3 shows the squared moduli of the wave functions, in free space $\left|\psi_{\mathrm{i}}+\psi_{\mathrm{r}}\right|^{2}$ and within the barrier, $\left|\psi_{\mathrm{tr}}\right|^{2}$, for $\mathrm{B}_{\max }=370$ Gauss, $\mathrm{v}_{0}=4 \mathrm{~m} / \mathrm{s}, \tau_{1}=0.6 \mu \mathrm{s}, \Lambda=2$ $\mu \mathrm{m}, \mathrm{a}=0.95 \mathrm{k}_{0}$. Within the barrier, a negative refraction effect brings the wave packet back to the edge. Note the (transient) narrowing of the transmitted wave packet. It is produced by the time-reversal property of the meta-medium [13]. In the free half-space $\mathrm{x}<0$, a rebound of the wave packet is seen at the same time as that observed within the barrier $(\mathrm{t} \approx 0.2 \mu \mathrm{s})$, as expected from the continuity conditions at the boundary. As a consequence the wave packet finally reflected at this time is retarded with respect to that obtained in absence of comoving pulse. Other features appear when the pulse duration is increased.
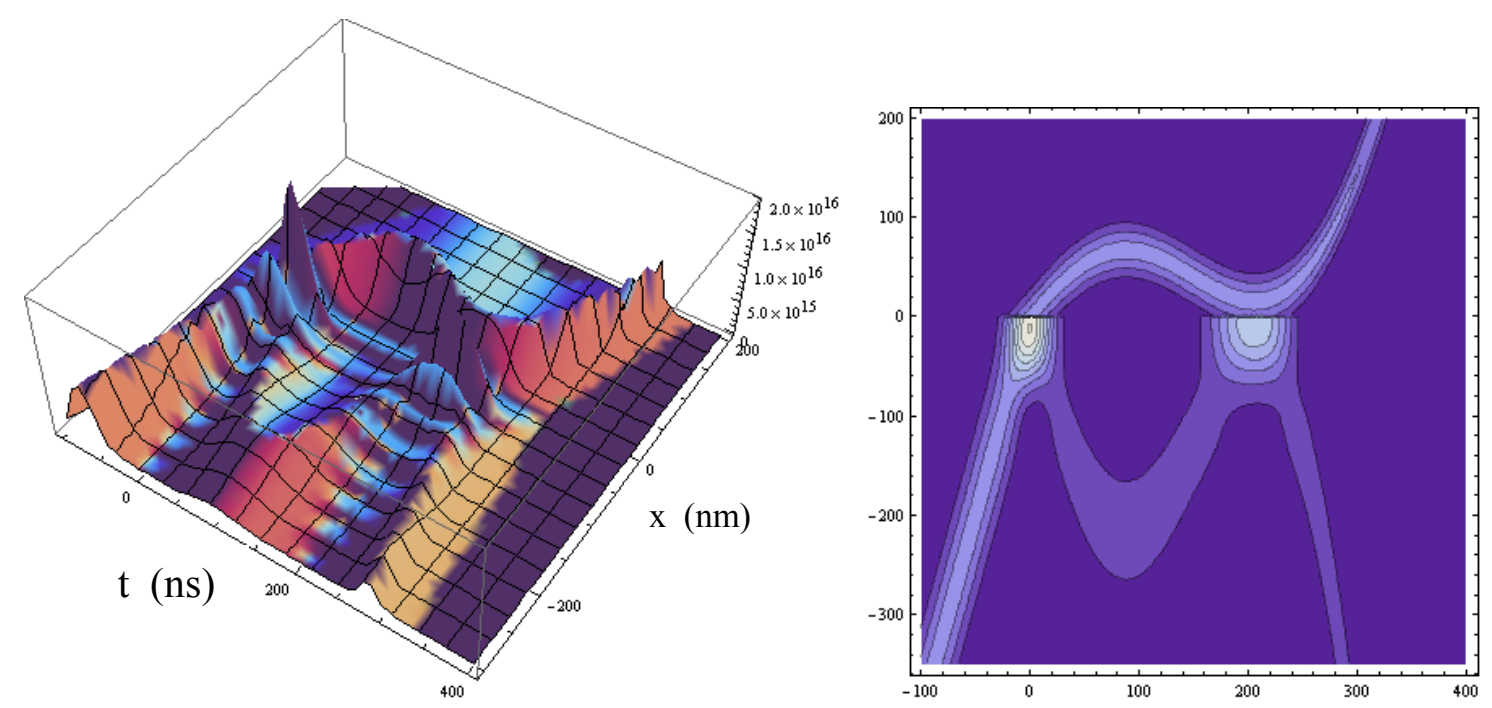

Figure 3. Profiles in $t$ and $x$ (3D surface and contour plot) of the squared modulus $\left|\psi_{\text {tr }}\right|^{2}$ of the transmitted wave function (in the $\mathrm{x}>0$ half-space) and $\left|\psi_{\mathrm{i}}+\psi_{\mathrm{r}}\right|^{2}$ of the total wave function in the $\mathrm{x}<0$ half-space, when a comoving pulse is applied. The parameters (see text) are as follows: $\mathrm{v}_{0}=4 \mathrm{~m} / \mathrm{s}, \mathrm{a} / \mathrm{k}_{0}=0.95, \Lambda=2 \mu \mathrm{m}, \tau_{1}=0.6$ $\mu \mathrm{s}, \mathrm{B}_{\max }=370$ Gauss. A negative refraction of the transmitted semi-evanescent wave is seen. It generates a rebound in the free half-space $(\mathrm{x}<0)$ and a retardation of the reflected wave packet.

Figure 4 shows the squared modulus of the wave functions on both sides of the barrier edge, under the following conditions: $\mathrm{B}_{\max }=150$ Gauss, $\mathrm{v}_{0}=4 \mathrm{~m} / \mathrm{s}, \tau_{1}=1.1 \mu \mathrm{s}, \Lambda=3 \mu \mathrm{m}, \mathrm{a}=1.01$ $\mathrm{k}_{0}$. On the positive-x side, the rebound previously seen for semi-evanescent wave packets is replaced by two evanescent contributions, one appearing at $\mathrm{t}=0$, the other one at $\mathrm{t} \approx 0.43 \mu \mathrm{s}$. In the free half-space, a real rebound connects these two times and the finally reflected wave packet is delayed to by the same amount of time with respect to the ordinary reflected wave packet. 


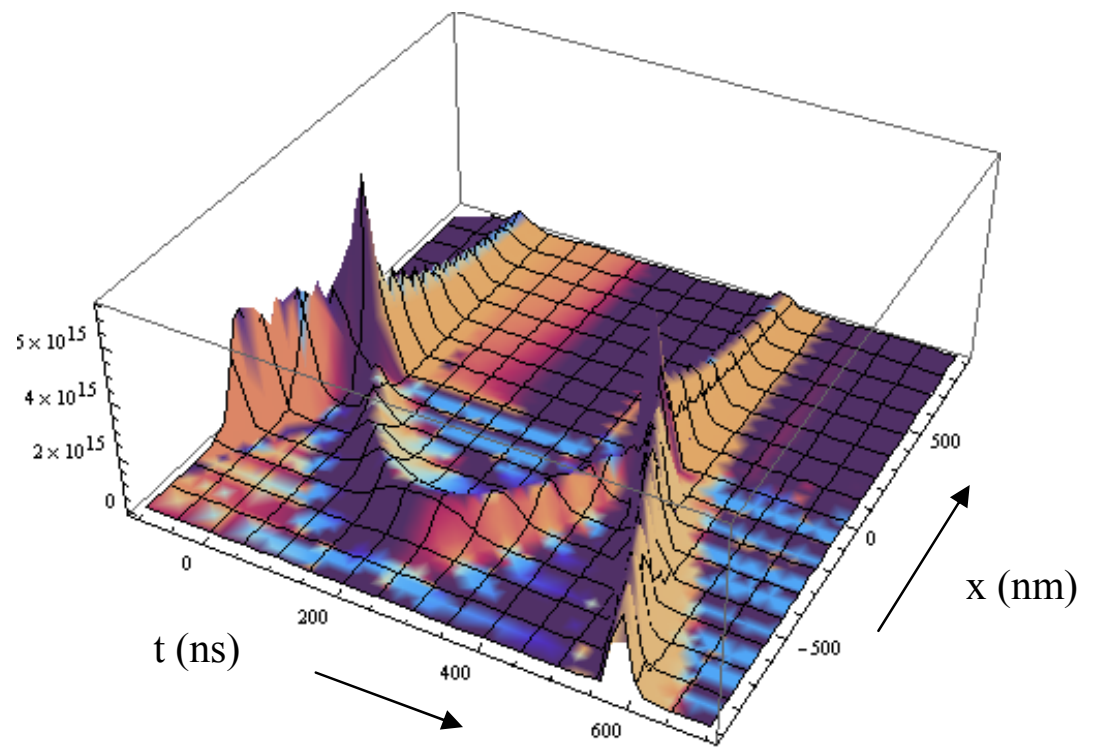

Figure 4. Same as fig. 3 (3D surface), with an almost purely evanescent wave (barrier height: $\mathrm{a}=1.01 \mathrm{k}_{0}$ ). The comoving pulse parameters are as follows: $\mathrm{v}_{0}=4 \mathrm{~m} / \mathrm{s}, \Lambda=3 \mu \mathrm{m}, \tau_{1}=1.1 \mu \mathrm{s}, \mathrm{B}_{\max }=150$ Gauss. An evanescent "echo" delayed by $0.43 \mu \mathrm{s}$ is generated within the barrier, whereas a rebound is seen in the free half-space causing a retardation of the finally reflected wave packet by the same amount of time.

Repeated similar effects are observed when two additional pulses, delayed by $0.605 \mu \mathrm{s}$ and $1.210 \mu \mathrm{s}$, are applied (see fig. 5). This is a clear evidence for the beginning of a temporal series of collapses and revivals of the evanescent matter wave at the potential barrier, accompanied by rebounds on the negative-x side. The same type of result is obtained with almost purely evanescent wave packets. This behaviour of the wave packets when subsequent pulses of comoving potential are applied strongly suggests the existence of a sort of a surface matter wave, which remains confined on both sides of the barrier edge ( $\mathrm{x}=0$ axis) while exhibiting a series of temporal rebounds or echoes along the $t$ axis. The related delay on the reflected wave packet should be a signature of the behaviour of the evanescent wave packet inside the barrier. Another observation mode would consist in cutting off the potential barrier at some distance, smaller than or equal to the evanescent decay length, and measuring the transmitted intensities. 


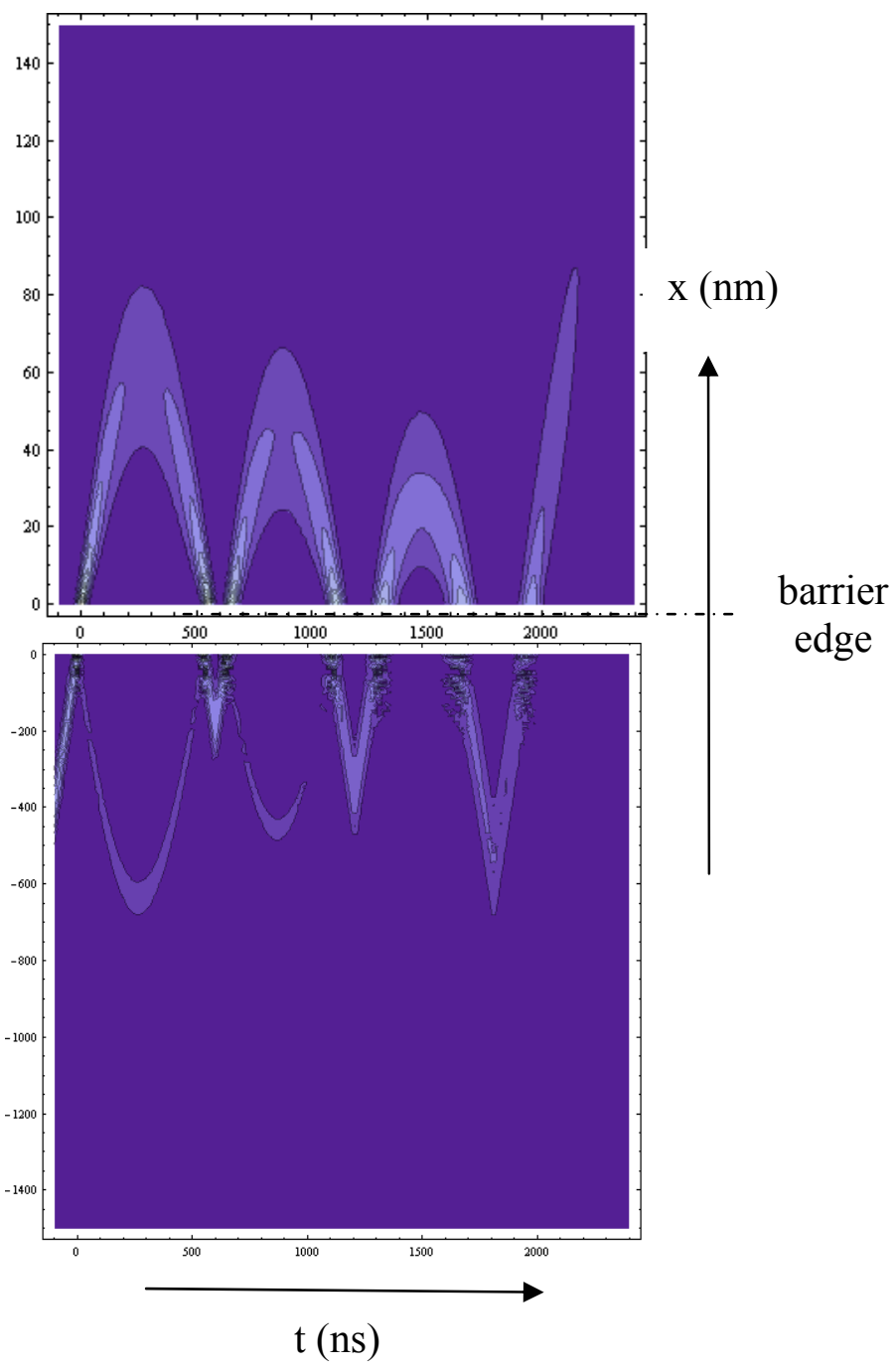

Figure 5. Same profiles as in figs 3, 4 (contour plot). A sequence of three identical comoving potential pulses is applied. Numerical parameters are as follows: $\mathrm{v}_{0}=4 \mathrm{~m} / \mathrm{s}, \mathrm{a} / \mathrm{k}_{0}=0.9975$ (semi-evanescent transmitted wave), $\Lambda=$ $3 \mu \mathrm{m}, \tau_{1}=0.6 \mu \mathrm{s}, \mathrm{B}_{\max }=120$ Gauss. Second and third pulses are delayed by $0.605 \mu \mathrm{s}$ and $1.210 \mu \mathrm{s}$ with respect to the first one. Note the difference by a factor 10 in $\mathrm{x}$-scales in regions $\mathrm{x}>0$ and $\mathrm{x}<0$.

To summarize, adequate pulsed comoving potentials have been proven not solely to induce a negative refraction of matter waves, but also to cause drastic effects on the dynamics of evanescent or semi-evanescent matter waves in a static potential barrier. It generates repeated enhancements, giving rise to a guided atomic "surface" wave, confined within an interval smaller than $1 \mu \mathrm{m}$ on both sides of the barrier edge and resulting into a retarded reflection. This makes these "negative index media" a counterpart of left-handed media in light optics, with however important differences related to the different natures of matter and light waves. Various applications can be imagined for matter waves. Some of them are similar to those of left-handed materials in light optics, such as atom wave focusing (meta lenses and atom nano- lithography), cloaking, etc. Some others are specific of matter waves such as beam splitters and interferometers and guided matter wave along a potential barrier edge. In this letter, we have mainly considered the special case of atoms with spin experiencing magnetic comoving potential pulses. Actually any type of potential possessing the same spatio-temporal characteristics as the present comoving pulses would induce effects quite similar to those described here. As an example we could envision electric comoving potential pulses acting on dipolar molecules and Rydberg atoms or molecules [14]. 
Authors are members of the Institut Francilien deRecherche sur les Atomes Froids (IFRAF).

\section{REFERENCES}

[1] See for instance: P. Meystre, Atom Optics, Springer (2001) [Series: At. Opt. Plas. Phys], and references therein

[2] F. Perales et al., Europhys.Lett. 78, 60003 (2007)

[3] V.G. Veselago, Sov. Phys. Usp., 10, 509 (1968)

[4] J. Pendry, Phys. Rev. Lett., 85, 3966 (2000); C. Foteinopoulou et al. Phys. Rev. Lett., 90, $107401(2003)$

[5] J. Baudon, M. Hamamda, J. Grucker, M. Boustimi, F. Perales, G. Dutier, M. Ducloy, Phys.Rev.Lett. 102, 140403 (2009)

[6] R. Mathevet et al. Phys. Rev. A, 56, 2954 (1997); M. Boustimi et al. Phys.Chem.News, 4, 1 (2001)

[7] G. Binnig, H. Rohrer, IBM Journal of Research and Development, 30, 4 (1986)

[8] B. D. Josephson, Rev. Mod. Phys. 46(2), 251 (1974)

[9] J.Levin et al. , Hyperfine Interactions, 127, 267(2000)

[10] See for instance Ch.J. Joachain, in Quantum Collision Theory, Elsevier Science ltd (1984)

[11] T. Schumm et al. Nature Physics, 1, 57 (2005)

[12] Such a short spatial period might be difficult to implement with a magnetic field. A different approach should be to use a near-resonant quasi-standing light wave.

[13] Narrowing of the matter-wave packet in negative-index media is a general feature of atom "meta-optics", which will be presented in detail in a forthcoming publication.

[14] H.L. Bethlem et al., Phys. Rev. Lett., 83(8), 1558 (1999); N. Vanhaecke et al., J. Phys. B, At. Mol. Opt.Phys. 38, S409 (2005) 